\title{
The heliospheric soft X-ray emission pattern during the ROSAT survey: Inferences on Local Bubble hot gas
}

\author{
R. Lallement ${ }^{\star}$
}

\author{
Service d'Aéronomie du CNRS, 91371 Verrières-le-Buisson, France
}

Received 17 April 2003 / Accepted 13 January 2004

\begin{abstract}
Solar wind interaction with neutral gas has been shown to generate soft X-rays, through the charge-exchange of highly ionized ions with neutral atoms and molecules in the interplanetary space and in the geocorona. The resulting diffuse emission can explain the non-cosmic long term enhancements (LTE) of ROSAT soft X-ray observations, which are produced by strong solar wind flux increases.

This paper focuses on the emission pattern resulting from the impact on the interstellar gas of the average, "quiet" solar wind, i.e., the non variable signal "below" the LTEs. A sky map of the heliospheric emission is calculated, based on realistic hydrogen and helium atom distributions under the influence of solar conditions such as those prevailing during the ROSAT survey. It is shown that parallax effects linked to the ROSAT observing strategy have a strong influence on the resultant emission pattern. For a stationary solar wind the modeled emission has an anisotropy factor of $\simeq 1.8$, and the emission maxima do not coincide with the interstellar wind axis direction. This emission is compared with the fraction of the ROSAT $0.25 \mathrm{keV}$ emission assigned to hot $\left(T=10^{6} \mathrm{~K}\right)$ gas in the Local Interstellar Bubble and with the dense gas contour maps of the Bubble drawn from $\mathrm{NaI}$ absorption. Assuming constancy of the global pattern, but allowing for a variable scaling factor, the maximum heliospheric contribution is derived and this allows the placement of lower limits on the truly interstellar emission in many directions. After subtraction of the heliospheric emission, there is significant interstellar $0.25 \mathrm{keV}$ emission from the Local Bubble "openings" to the lower halo, as expected, but also to neighbouring interstellar "bubbles", as well as towards a number of regions along the galactic plane. There is evidence that there is hot gas emission attributable to our local cavity and generated throughout its volume, including along the galactic plane, but significantly weaker than initially thought. This would attenuate the discrepancy by a factor of about $4-5$ between the hot gas pressure initially derived without heliospheric decontamination and the pressure of the embedded local diffuse clouds.
\end{abstract}

Key words. ISM: clouds - ISM: atoms - Galaxy: solar neighborhood

\section{Introduction}

After the surprising discovery of X-ray emission from comet Hyakutake (Lisse et al. 1996), a number of other comets have been found to emit X-rays. Among the mechanisms invoked concerning the origin of the emission, charge transfer of heavy solar wind ions with cometary neutrals, first proposed by Cravens (1997), seems to be the most reasonable. This solar wind charge exchange (SWCX) has been shown to reproduce the observed emission features (Häberli et al. 1997; Krasnopolsky 1997; Kharchenko \& Dalgarno 2000; Schwadron \& Cravens 2002).

As with cometary neutrals, neutral atoms of interstellar origin in the solar system should also experience the SWCX reactions, and the de-excitation of charge-exhanged ions should generate diffuse soft X-ray emission throughout the whole heliosphere, as suggested by Cox (1998), and first modeled by Cravens (2000).

The soft X-ray diffuse background has been successfully mapped by the Position Sensitive Proportional Counter (PSPC)

* e-mail: rosine.lallement@aerov.jussieu.fr of the ROSAT X-ray telescope (Snowden et al. 1995, 1998). While the $0.25 \mathrm{keV}$ band primarily traces emission from local hot interstellar gas, the total observed flux has been interpretated as the sum of three physical components, i.e., extragalactic, halo and Local Bubble (LB) hot gas contributions. Details of these different models and the constraints on the location of the soft X-ray emitting gas deduced from a number of soft X-ray shadows can be found in Snowden et al. $(1998,2000)$, and Kuntz \& Snowden (2000). From these data the temperature of the local hot gas has been found to be of the order of $10^{6} \mathrm{~K}$. If the gas which is filling the LB is assumed to be homogeneous, the measured emission in each direction is proportional to the path length through the hot gas up to the region of dense and cool gas, and therefore provides a mapping of the LB itself. The resulting shape of the emitting region has been derived by Snowden et al. (1998), and among the main features are two large elongations towards opposite directions at high galactic latitudes. Their axis is tilted by about $20-30^{\circ}$ with respect to the galactic polar axis, and they have been shown to coincide with neutral gas-free "chimneys", nearly perpendicular to the Gould belt (Sfeir et al. 1999; Welsh et al. 1999). 
During the ROSAT survey, sporadic increases of the count rate lasting for a few hours to a few days were observed repeatedly. These "long-term enhancements" (LTEs) have been shown to be correlated with the solar activity (Freyberg 1994), and more precisely with the solar wind events (Cravens 2000). It is now evident, after the convincing study of Cravens et al. (2001) and Robertson et al. (2001), that they are mainly due to enhanced SWCX in the interplanetary medium, responding to strong solar wind events such as CMEs. The geocorona itself adds a significant contribution to the LTE signal. Cravens et al. (2001), using a simple model of neutral gas distribution and solar wind data, have successfully reproduced the temporal behavior of the LTEs. But, if solar wind enhancements produce the LTEs, there is also a "quiet" heliospheric emission associated to the "normal" or "quiet" solar wind, in the absence of strong flux enhancements. In other words, LTEs are the visible part, on top of an underlying quasi stable background. Cravens et al. (2001), using updated cross-sections and their simplified model of interstellar distribution, have modeled the total emission, i.e., emission generated by all the solar wind, in the direction of the incoming interstellar wind (upwind), and find $4 \pi I \approx 90 \mathrm{keV} \mathrm{cm} \mathrm{cm}^{-2} \mathrm{~s}^{-1}$ for the hydrogen emission, which is the dominant contribution in this direction and about $140 \mathrm{keV} \mathrm{cm} \mathrm{s}^{-1}$ for the total emission. This translates into $I \approx 30 \mathrm{keV} \mathrm{cm} \mathrm{cm}^{-2} \mathrm{~s}^{-1} \mathrm{sr}^{-1} \mathrm{keV}^{-1}$ assuming a thermal spectrum at an effective temperature of $0.2 \mathrm{keV}$, which is a reasonable hypothesis according to the spectral measurements of comet emissions (Dennerl et al. 1997). A first map of the emission associated with charge exchange with helium and hydrogen has been drawn by Robertson et al. (2001) and displays an axisymmetric pattern with a maximum to minimum ratio of about 2, with a broad maximum on the upwind side and a secondary maximum on the downwind side. This map corresponds to a unique location of the spacecraft on the upwind side. Another way to estimate the contribution of the "quiet" heliospheric emission is the derivation of a global scaling factor linking the solar wind flux and the SWCX emission from the adjustment of the LTE levels to the solar wind flux enhancements (Cravens et al. 2001). This, in turn, allows the estimation of the contribution from the quasi-stationary solar wind. In doing so, Cravens et al. (2001) calculate similar numbers to those derived from their modelling and updated cross-sections. Because the derived heliospheric intensity is of the same order as the $25-80 \mathrm{keV} \mathrm{cm}{ }^{-2} \mathrm{~s}^{-1} \mathrm{sr}^{-1} \mathrm{keV}^{-1}$ intensity in the $0.25 \mathrm{keV}$ band of ROSAT attributed up to now to the local hot gas emission (Snowden et al. 1998), and actually includes both emission from hot gas in the Local Bubble and emission from the heliosphere, the question of the remaining truly LB emission then arises naturally. Since there are a number of unexplained observations about the hot gas and the ionization in the local bubble and the local clouds (e.g., Breitschwerdt et al. 2000; Breitschwerdt 2001), this question deserves more investigation. We start with a comparison of the emission patterns (which is the main goal of this paper) and also estimate quantitatively the intensity, using a number of assumptions.

Solar wind ion impacts on neutral hydrogen and helium are the dominant reactions in the heliosphere. The spatial distribution of those two species are very different and need to be modeled independently. Here, I use a model of the interstellar helium flow recently improved for the analysis of $\mathrm{SOHO} /$ UVCS and EUVE observations (Lallement et al. 2003a,b), as well as a model of interstellar hydrogen used for the analysis of SOHO/SWAN data (Costa et al. 1999). Neutral atom distributions appropriate to the time of the ROSAT survey (1990-1991) are computed within $150 \mathrm{AU}$. The resulting emission pattern is derived from those distributions according to the observing geometry of the survey, assuming an isotropic quiet solar wind. It is essential to take into account this geometry, because the solar system X-ray emission depends significantly on the position of the observer. This emission pattern is then compared with the fraction of the ROSAT $0.25 \mathrm{keV}$ emission attributed to the local hot gas, which includes hot gas from the Local Bubble and heliospheric emission, taken from Snowden et al. (1998). It is important to note that, for those data, the LTE contribution has been already removed by the authors. This means that, if, ideally, the solar wind can be considered as the sum of a constant and isotropic background flux, plus sporadic enhancements, those events have already been taken into account. We will discuss this hypothesis in the last section. Here, the geocoronal stationary emission is neglected. According to Cravens (2001), and at variance with the geocoronal contribution to LTEs, this emission amounts to only a few percent of the total "solar system" emission (Lallement et al. 2003c).

Results of the comparison are shown in the galactic plane, and vertical planes, and compared with the dense neutral gas boundary to the Local Bubble as recently mapped from interstellar NaI spectra taken towards nearby stars. Residual intensities are also shown within two assumptions on the general intensity level.

\section{The heliospheric model for hydrogen and helium}

The $\mathrm{H}$ and $\mathrm{He}$ densities are computed using so-called "hot model" type codes, which follow individual trajectories of neutral atoms in the heliosphere. The parameters of the helium flow are now very well defined (Witte et al. 2003) and here taken as: $V(\mathrm{He})=25.5 \mathrm{~km} \mathrm{~s}^{-1}, T(\mathrm{He})=7000 \mathrm{~K}, \lambda=74.5^{\circ}, \beta=-6.5^{\circ}$ (ecliptic coordinates of the helium velocity vector). The solar photoionisation rate of helium at the time of ROSAT observations, i.e., August 1990 to January 1991, is estimated using a proxy based on the Magnesium index, recently updated to fit SOHO SEM observations (McMullin et al. 2002): $\beta_{\text {phot }}=$ $0.91 \times 10^{-7} \mathrm{~s}^{-1}$ at $1 \mathrm{AU}$ The rate for electron impact ionisation is estimated by analogy with the UVCS based rates of Lallement et al. (2003b) of the last solar cycle, using numbers for the same solar activity phase, or twice the average level of Rucinski \& Fahr (1988).

The hydrogen flow parameters are those derived from SOHO/SWAN hydrogen cell results (Costa et al. 1999; Lallement 1999), i.e., $V(\mathrm{H})=22 \mathrm{~km} \mathrm{~s}^{-1}, T(\mathrm{H})=11000 \mathrm{~K}$, which apply to the gas after entrance in the heliosphere, i.e., after deceleration and heating through coupling with the piling up interstellar plasma. The present hydrogen model does not include the density increase at the periphery of the heliosphere, however this increase has a very small effect on 
the resulting integrated emission, because the emissivity decreases strongly with distance. The ionisation rate of hydrogen is slightly latitude dependent, with $\beta=0.9 \times 10^{-6} \mathrm{~s}^{-1}$ at $1 \mathrm{AU}$ along the solar equatorial plane, $\beta=0.8 \times 10^{-6} \mathrm{~s}^{-1}$ between 10 and 70 degrees heliolatitude, and $\beta=0.7 \times 10^{-6} \mathrm{~s}^{-1}$ above $70^{\circ}$ heliolatitude. The ratio of Lyman-alpha radiation pressure to gravitation is taken to be 0.90 , as found in 1996 from the SOHO-SWAN Hydrogen cell analysis (Costa et al. 1999). This value may be too low for a high activity period as in 1990, but its choice is motivated by past observations of backscattered solar radiation which have shown that distributions calculated for much larger ratios underestimate the filling of the hydrogen ionisation cavity, something not satisfyingly explained yet.

In the charge-exchange process, the resulting soft X-ray total emissivity at distance $r$ from the Sun is proportional to the solar wind flux $u_{\mathrm{sw}} n_{\mathrm{sw}}(r)$ and the neutral atom density $n_{(\mathrm{H}, \mathrm{He})}(r)$. Assuming a homogeneous solar wind, i.e., constant ion relative abundances, one can follow Cravens (2000) and use a global efficiency or cross section $\alpha$. The emission is the integral along the line-of-sight (LOS) of this emissivity:

$4 \pi I=\alpha \int_{\text {earth }}^{\infty} n(r) u_{\mathrm{sw}}(r) n_{\mathrm{sw}}(r) \mathrm{d} r$.

Thus $4 \pi I=\alpha u_{\mathrm{sw}}^{\circ} n_{\mathrm{sw}}^{\circ} \int_{\mathrm{earth}}^{\infty} n(r) r^{-2} \mathrm{~d} r$ where $u_{\mathrm{sw}}^{\circ} n_{\mathrm{sw}}^{\circ}=F_{\mathrm{sw}}^{0}$ is the solar wind particle flux at $1 \mathrm{AU}$.

\section{Results for the ROSAT survey observing strategy and solar activity phase}

Because the emissivity maxima are found at distances of the order of $1 \mathrm{AU}$, or even much smaller in the case of helium, the above integral depends on both the distributions and the observer (spacecraft) location. For this reason the emission pattern of $\mathrm{H}$ and $\mathrm{He}$ as seen by ROSAT during the 6 months survey differs significantly from the pattern seen by an observer located at the Sun. While the latter is not very far from axisymmetric around the Sun-wind direction, the former is far from being axisymmetric. As a matter of fact, ROSAT survey data were acquired in the spinning mode with the field-of-view perpendicular to the rotation axis (Snowden et al. 1994), and with the spin axis constrained to the ecliptic plane with an angle $\leq 15^{\circ}$ from the Sun-Earth line. More precisely, the satelite spin axis, which was perpendicular to its look direction, was oriented roughly towards the Sun and kept at an ecliptic longitude slightly larger than the Sun longitude. This was for safety reasons linked to the spacecraft operations. This means that at a given ecliptic longitude of the direction of sight does correspond a unique date of observation, $\approx 3$ months before or after the earth was at this longitude. A "ROSAT" specific map has to be calculated by assigning at each sightline its date and earth location. In the calculations we have assumed that the spin axis was at an ecliptic longitude larger by a constant angle of $15^{\circ}$ than the Earth-Sun line. Errors on the spin axis direction are always smaller than $4^{\circ}$.

A very interesting aspect of the SWCX mechanism is its dependence on the solar wind type, mainly because high charge state ion abundances differ strongly between the slow wind, the fast wind and CME's. Kharchenko \& Dalgarno (2000) have calculated fast and slow solar wind spectra, and it is clear from their study that intensities may be significantly different, especially in the $3 / 4 \mathrm{keV}$ energy range. In the case of the ROSAT survey, the solar wind was characterized, as it does at every maximum of activity, by very small or nonexistent polar coronal holes, and ubiquity of slow type solar wind. On the other hand, coronal mass ejections, which are also frequent at high solar activity, are not so important here, since they correspond to strong flux enhancements and they have been included in the LTE removal. As a conclusion, the assumption of solar wind isotropy, at least for the present estimate of the background solar wind charge-exchange, is reasonable. Figure 1 shows the resulting sky emission separately for hydrogen and helium, and the total emission from both gases in galactic coordinates. The so-called upwind and downwind directions are indicated. The helium map shows clearly strong departures from an axisymmetric pattern such as an observer at the Sun would see, with a maximum along the interstellar wind axis in the downwind direction. In particular there are enhancements towards high Galactic latitudes, both north and south. These departures are less conspicuous for hydrogen, due to the strong ionization of this gas for the maximum activity period in 1990-91 which has the effect of increasing the size of the empty cavity around the Sun, and minimizes the parallax effects. This is also also due to the small anisotropy of the solar wind at maximum activity. This would be significantly different for minimum activity.

Quantities plotted in the maps of Fig. 1 are the integrals $\int_{\text {earth }}^{\infty} n(r) r^{-2} \mathrm{~d} r\left(\mathrm{~cm}^{-3} \mathrm{AU}^{-1}\right)$, converted into intensities $4 \pi I$ then to ROSAT units after multiplication by a global factor 8050 , which is justified in the next section. The minimum and maximum count rates are found to be about 240 and $450 \times$ $10^{-6}$ counts s$^{-1}$ arcmin $^{-2}$ resp. Surprisingly, this diffuse emission due to the background solar wind is not very strongly anisotropic, with a factor of anisotropy of only 1.8 for the updated interstellar and solar parameters used here. The differences between the predicted pattern and the first map of Robertson et al. (2001) are due mainly to the differences in the observing conditions, and in the distributions of the interstellar gases. The order of magnitude of the maximum intensity is however similar.

\section{Comparison with the ROSAT local gas emission maps}

The conversion of the integrated emissivities into ROSAT units is made in a simplified way as follows. First, we use a global cross-section $\alpha=7.5 \times 10^{-16} \mathrm{eV} \mathrm{cm}^{2}$ (Schwadron \& Cravens 2000; Wegmann et al. 1998; Kharchenko \& Dalgarno 2000), which includes all the physics of the charge-exchange mechanism and relative abundances, and a constant and isotropic solar wind flux $F^{0}=3.0 \times 10^{8} \mathrm{~cm}^{-2} \mathrm{~s}^{-1}$, the classical average solar wind flux. The actual values of the cross-sections are the subject of considerable work, but due to the large number of contributing ions, the differences between the different solar wind regimes, there are still significant uncertainties on them. 

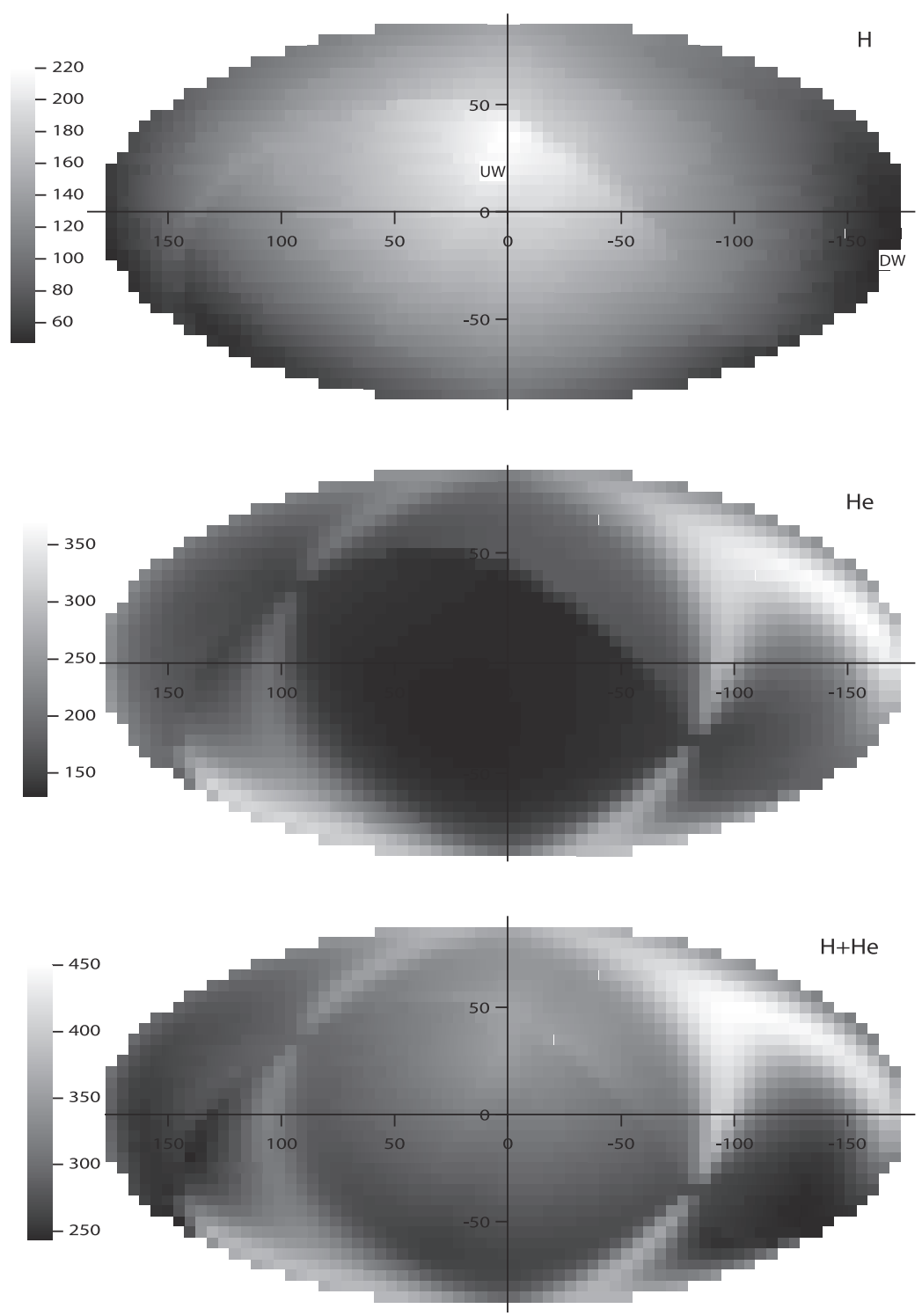

Fig. 1. Maps of the model emission for H, He, and their total contribution. Units are similar to those used by Snowden et al. (1998), i.e., $10^{-6}$ counts $\mathrm{s}^{-1} \operatorname{arcmin}^{-2}$ of the ROSAT PSPC R1+R2 band. Conversions have made under the assumptions described in the text. UW and DW mark the directions of the incoming interstellar wind (upwind), and its opposite (downwind). The discontinuity is due to the observing geometry and the fact that two adjacent lines-of-sight may have been observed 6 months apart, which changes the parallaxes. The images use an Aitoff projection in zero-centered galactic coordinates with longitude increasing to the left.

In addition, the role of the neutral target is still debated. This is why, in the following, cross-sections for $\mathrm{H}$ and $\mathrm{He}$ are assumed to be identical.

Then, in order to convert the total energy into a spectral energy at $0.2 \mathrm{keV}$, we use the same assumption on the spectral shape as Cravens et al. (2000), i.e., a thermal spectrum at $0.2 \mathrm{keV}$, and the resulting relationship: a spectrally integrated intensity $I=11 \mathrm{keV} \mathrm{cm} \mathrm{cm}^{-1} \mathrm{sr}^{-1}$ corresponds to $31 \mathrm{keV} \mathrm{cm}^{-2} \mathrm{~s}^{-1} \mathrm{sr}^{-1} \mathrm{keV}^{-1}$ at $0.2 \mathrm{keV}$. Finally, the ROSAT PSPC sensitivity, discussed in Snowden et al. (1998), is such that in the $R 1$ and $R 2$ bands a count rate of $10^{-6}$ counts $\mathrm{s}^{-1} \operatorname{arcmin}^{-2}$ corresponds to the unabsorbed emission of gas at $10^{6} \mathrm{~K}$ in thermal equilibrium (Raymond \& Smith 1977) at a level of $0.1 \mathrm{keV} \mathrm{cm} \mathrm{cm}^{-2} \mathrm{~s}^{-1} \mathrm{sr}^{-1} \mathrm{keV}^{-1}$ at $0.2 \mathrm{keV}$. Those steps, when combined, imply that the integrals $\int_{\text {earth }}^{\infty} n(r) r^{-2} \mathrm{~d} r\left(\mathrm{~cm}^{-3} \mathrm{AU}^{-1}\right)$ are roughly converted into ROSAT units (i.e., $10^{-6}$ counts $\mathrm{s}^{-1} \operatorname{arcmin}^{-2}$ ) by multiplying by a global scaling factor of $\simeq 8050$ ROSAT Units $\mathrm{cm}^{3}$ AU. Evidently, uncertainties on this global scaling are significant, the main sources being the uncertainty of the chargeexchange-deexcitation cross-section, the spectral shape of the emission, and also the fraction $k$ of nominal solar wind, here defined as the fraction which remains after subtraction of all the events having been already interpreted as LTEs and removed. The $k=1$ scaling used in the maps corresponds to the high 
contamination case, meaning that only enhancements due to the geocorona, i.e., those conspicuous spikes shown by Cravens et al. (2001), have been removed from the ROSAT data. This probably overestimates the value of the solar wind flux appropriate for the cleaning of the data, but not by more than a factor of two (Snowden 2003).

These emission levels are shown in several planes, the galactic plane (Fig. 2a) and 6 vertical planes crossing the galactic plane every 30 degrees (Fig. 3), the first plane being the meridian plane defined by the galactic center and the polar axis. Again, the total $\mathrm{H}+\mathrm{He}$ emission due to the background solar wind is surprisingly regular. Superimposed on this are drawn the ROSAT unabsorbed local emissions derived by Snowden et al. (1998), in the same units. These intensities have been carefully cleaned from LTEs by these authors, who have used most of the time the redundancy of the observations during multiple rotations (The ROSAT All-Sky survey scanned a great circle on the sky every orbit with the plane of the great circle precessing by $\simeq 4^{\prime}$ each orbit. The field of view of the PSPC was slightly less than $2^{\circ}$ diameter so the multiple coverage was extensive).

The heliospheric emission curves in the planes of Figs. 2a and 3 have been drawn in the maximum contribution case, which corresponds to the values from maps of Fig. 1 decreased by $10 \%$ (all intensities have been multiplied by $k=0.9$ ). This is the maximum possible heliospheric contribution case, because for values of $k$ larger than 0.9 , there are directions for which the model heliospheric emission becomes larger than the measured ROSAT rate. In other words, $k \leq 0.9$ ensures that the maximum value of the heliospheric background emission is always smaller than the ROSAT emission. The fact that the estimated average value of the heliospheric emission is similar to the maximum allowed emission suggests that indeed a large part of the emission measured by ROSAT can have its origin in the heliosphere, as predicted by Cravens et al. (2001).

Figures $2 \mathrm{a}$ and 3 , because they correspond to the maximum case, show the fraction of the soft X-rays which can not be attributed to the solar system. In order to investigate the source regions for these truly IS emissions, contours of the local cavity obtained from neutral sodium absorption mapping (Lallement et al. 2003c) are superimposed on the soft X-ray polar plots. The comparison between those contours and the difference between the ROSAT and the heliospheric emission is instructive. Interestingly, the largest discrepancies between the two emissions essentially arise in three types of regions: i) those for which the derived local emission is contaminated by background intense objects as Cygnus and Monogem, marked by shaded contours in the Snowden et al. maps, ii) high galactic latitudes, and iii) local cavity elongations.

In the galactic plane, if one omits the contaminated areas (ROSAT shaded contours), there are two regions characterized by LB elongations, and those two regions, at $l_{\mathrm{II}}=120^{\circ}$ and $330^{\circ}$ are also those with an excess of measured intensity wrt the maximum heliospheric emission. Assuming that all of the emission is of heliospheric origin towards the galactic center and the anticenter (the maximum contamination case), the excess is of the order of $25-35 \%$ of the total. This suggests that the true LB hot gas emission in those directions is at minimum one fourth of the initial contaminated value. In the maximum contamination case however, hot gas emission would arise essentially from those elongated parts of the local cavity.

In the meridian plane (Fig. 3) the comparison between the two emission patterns shows that there are very large excesses of measured intensity at high galactic latitude, from the two "chimneys" which characterize the local cavity shape in this plane and connect it to the lower halo. At least $50 \%$ of the emission is apparently due to hot gas from those elongations, which are inclined by $20-30^{\circ}$ with respect to the polar axis. Apart from that, there is an excess towards $l, b=180,-40$, may be related to the proximity of Eridanus region.

In the rotation plane, again omitting "difficult" areas, the intensity at high positive and negative latitudes is largely in excess, with up to at least 1.2 times the solar system emission. The two maxima at 90, 60 and 270, -60 however do not seem to correspond to LB elongations. In general, all vertical planes in Fig. 3 show that a large fraction of the emission is coming from high latitudes.

Figure $2 b$ shows the residual intensity, i.e., ROSAT measurement after subtraction of the heliospheric counterpart, again in the galactic plane, for two cases, i) $k=0.9$, the maximal case, and ii) for $k=0.7$, i.e., assuming the global intensity level of the heliospheric intensity is $70 \%$ of the "nominal solar wind" value, which is more likely than $k=1$ (see discussion). In the former case, the residual intensity is by definition zero in some directions, while in the latter it is positive in all directions. Interestingly, the intensity pattern in this latter case bears some resemblance with the cavity contours in the uncontaminated areas, as would be the case if the emission were generated throughout the volume of the cavity. If confirmed, this would imply that there is indeed hot gas within the local cavity, including along the galactic plane, at a pressure several times smaller (about 2 times as suggested by Fig. 2b, see discussion below) than the pressure previously derived when all the X-ray emission was assigned to an emitting, hot million $\mathrm{K}$ interstellar gas.

\section{Discussion}

The quantitative comparison between the modeled heliospheric soft X-ray background in the nominal solar wind regime and the unabsorbed fraction of the soft X-ray $0.25 \mathrm{keV}$ emission confirms that a significant fraction of the latter could be of heliospheric origin, as predicted by Cox (1998), and Cravens (2000, 2001). The heliospheric pattern is significantly influenced by the earth location at the time of the obervation, and for this reason the ROSAT six months survey pattern is NOT axisymmetric and rather complex. Emission maxima are OUTSIDE the interstellar wind axis directions.

The amount of heliospheric emission which contaminates the ROSAT soft X-ray maps is the fraction of the total heliospheric contribution which has not been substracted during the process of LTEs removal. This fraction is treated here a global scaling factor $k$. Equivalently, $k$ is the fraction of the solar wind output which has not been already taken into account in the LTE removal process. $k=1$ corresponds to the nominal solar 

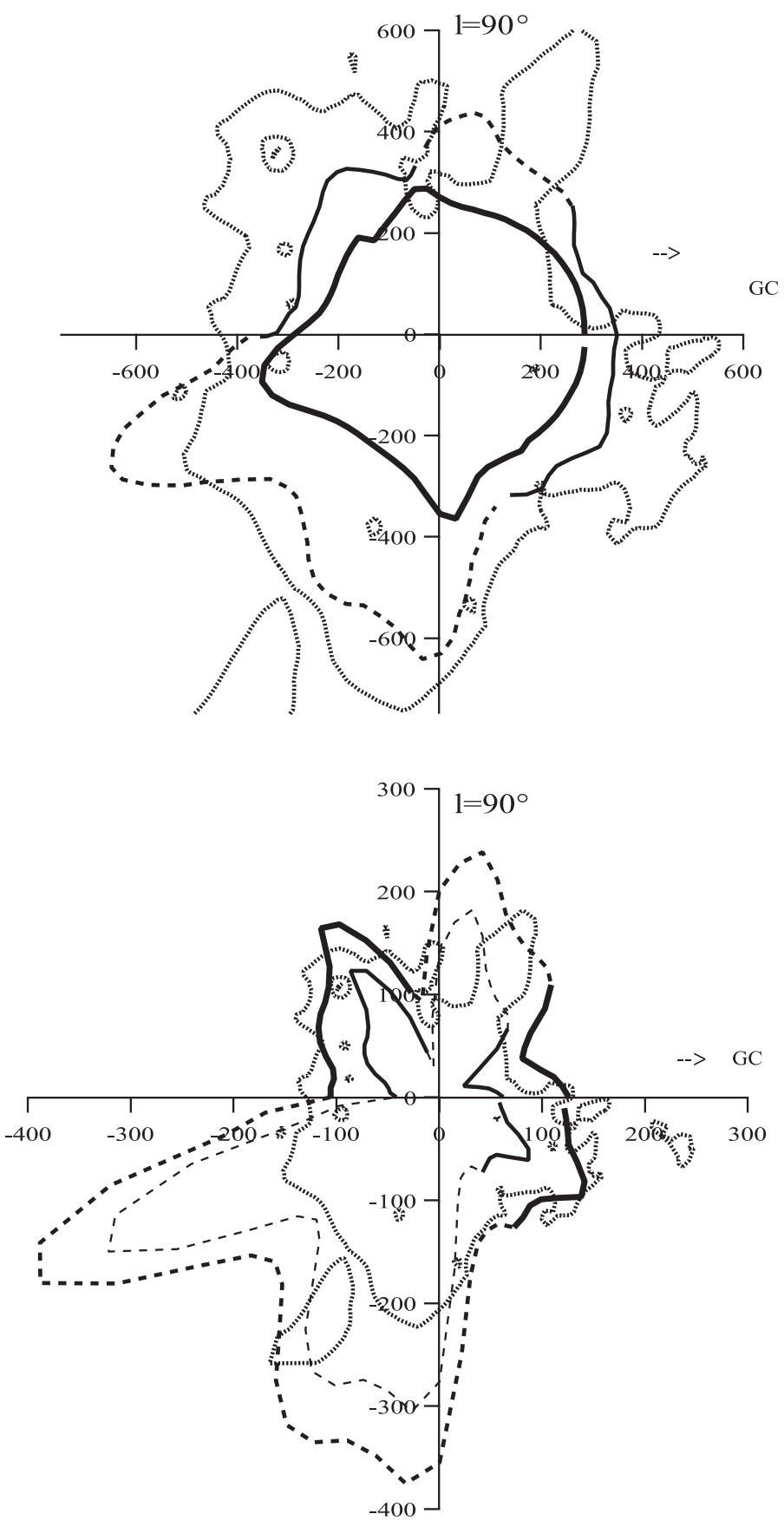

Fig. 2. Top: comparison between the model heliospheric emission (thickest line), the scaled ROSAT unabsorbed soft X-ray ( $0.25 \mathrm{keV})$ emission (thick solid line), and the neutral gas boundary to the local cavity derived from interstellar NaI absorption spectra (dotted line) in the galactic plane. The dashed lines for the X-ray data correspond to directions of bright distant objects, and thus to less precise determinations of the local emission (Snowden et al. 1998). Units for the soft X-ray emissions are those used by Snowden et al. (1998), i.e., $10^{-6} \mathrm{counts} \mathrm{s}^{-1} \mathrm{arcmin}^{-2}$ of the PSPC R1+R2 band. Units for the distance to the dense gas are parsecs multiplied by 10/3. The heliospheric intensity is the one of Fig. 1, multiplied by $k=0.9$, the highest scaling factor compatible with the data, i.e., ensuring the heliospheric intensity in all directions remains smaller than the measurements. In a number of regions the ROSAT emission is stronger or much stronger than the heliospheric emission, even in this "maximum" contamination case. Bottom: interstellar emission after subtraction of the model heliospheric counterpart, in the galactic plane: a) in the maximum contribution configuration $(k=0.9)$, which corresponds to the top figure (solid line); b) assuming $k=0.70$ (thick solid line). Units for the distances to the local cavity contours are parsecs. The LB boundary contours and case b emission patterns are roughly similar in the unambiguous regions, suggesting the latter could be the true ISM emission from hot gas filling the cavity. In this case, the intensity would be of the order of $25 \%$ of the total. 

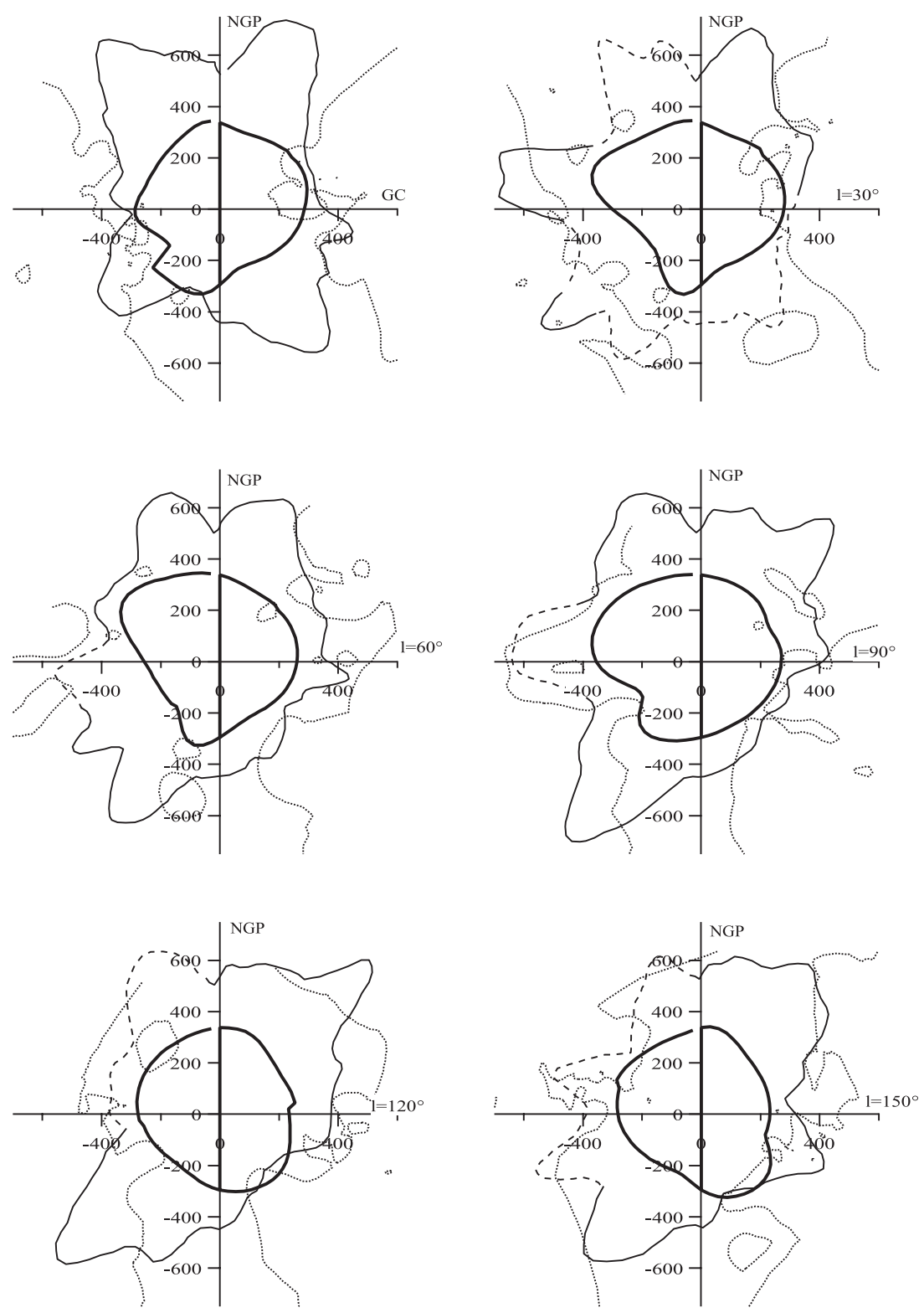

Fig. 3. Comparison between the model heliospheric emission (thickest line), the ROSAT unabsorbed soft X-ray (0.25 keV) emission (thick solid line), and the NaI local cavity contours (dotted line) in planes perpendicular to the galactic plane, every 30 degree longitude, starting from the meridian plane. Units are identical to Fig. 2, top. At high galactic latitude the emission is in average twice the maximum heliospheric contribution.

wind flux, and would imply that removed LTEs contained essentially the geocoronal response to the solar wind events, but only a negligible fraction of the interstellar gas contribution. It is very likely that $k \leq 1$, but a minimum value for $k$ is difficult to estimate since it depends on the LTE removal algorithm. In addition to the uncertainty on the actual value of this fraction $k$, there are large uncertainties on the derived emission level, linked to uncertainties on the cross-sections and on the chargeexchange spectrum. The comparison of the model heliospheric pattern and the observed X-ray maps allows to derive a maximum value of $k$, found to be of the order of 0.9 , and shows that in a number of directions the measured ROSAT emission exceeds significantly the maximum heliospheric contribution, in particular at high galactic latitudes, i.e. halo emission, but also in the galactic plane. In order to visualize this lower limit to the true interstellar hot gas emission, ROSAT measurements minus the maximum allowed heliospheric background are also shown in the galactic plane. In this "maximal contamination" case, there would be no hot gas emission in the galactic plane at the Sun location, all the emission would come from more distant regions, halo and adjacent cavities. Interestingly, the comparison with the local cavity dense gas contours shows a link between those regions with significant brightness excess on one hand, and local cavity elongations linking it to the halo and to neighbouring bubbles on the other. More work however is needed however to map both the dense and the diffuse gas in order to make those links clearer, and to compare with the soft $\mathrm{X}$-ray shadows. 
We also show the residual emission in the case $k=0.7$, which, according to uncertainties in the cross-sections and in the spectrum, is as probable as $k=1$, and is more likely from the point of view of the LTE removal (Snowden 2003). For $k=0.7$ (or an equivalent solar wind flux $F^{0}=2.1 \times$ $10^{8} \mathrm{~cm}^{-2} \mathrm{~s}^{-1}$ ), the emission pattern in the galactic plane has similarities with the cavity contour pattern, except for the areas known to be contaminated by bright objects beyond the local cavity (Snowden 1998). This is in agreement with the displacement model, i.e., hot gas emission generated throughout the LB volume. However in this case the hot gas pressure, derived from the ratio between the X-ray intensity and the path length through the hot gas, i.e., the distance to the LB boundary, is about 2.5 times lower than the one initially found. As a matter of fact, Snowden et al. (1998) derived an $\mathrm{X}$-ray scale factor of 0.155 , based on the estimated distance to the molecular cloud MBM12 and using total intensities, while here the scale factor is $\simeq 1$., using LB boundaries derived from absorption maps, and after removal of heliospheric contamination (for $k=0.7$ ). Instead of $n T=10000-15000 \mathrm{~K} \mathrm{~cm}^{-3}$ (e.g., Snowden et al. 1998; Burrows \& Guo 1998), the hot gas pressure could be as low as $4000-6000 \mathrm{~K} \mathrm{~cm}^{-3}$. In this case there would no longer be any strong difference between the LB hot gas pressure and the gas pressure in the Local Cloud surrounding the Sun ( $n T$ around $2300-2600 \mathrm{~K} \mathrm{~cm}^{-3}$, Lallement 1998) or in other diffuse clouds embedded in the cavity (Jenkins 2002), which would resolve a longstanding anomaly, in a less sophisticated way however than the new physical model recently devised by Cox \& Helenius (2003).

Whatever the actual level of the heliospheric contamination, it results in a lower value of the hot gas pressure and reduces the pressure difference. It may also explain the absence or very low amount of OVI ions supposedly formed at the interface between those diffuse clouds and the hot gas, as recently deduced from FUSE nearby white dwarf observations (Oegerle et al. 2000). Finally, it may explain the very low upper limit put by EUVE on the diffuse background measurements in the EUV (Vallerga \& Slavin 1998), and why soft X-ray spectra have weak iron emission lines (Mc Cammon et al. 2002). New EUV measurements with the large aperture of the NASA CHIPS microsatellite and new high resolution soft X-ray spectral data should help to establish the true level of the hot gas emission.

Acknowledgements. I thank Michael Freyberg for informations on Rosat, Tom Cravens and Ina Robertson, as well as Don Cox and Dan McCammon for useful discussions. I am very grateful to Steve Snowden, for his criticisms, comments, suggestions, and corrections which contributed to the clarity of the paper.

\section{References}

Breitschwerdt, D., Freyberg, M. J., \& Egger, R. 2000, A\&A, 361, 303 Breitschwerdt, D. 2001, Astr. Sp. Sci., 276, 163

Costa, J., Lallement, R., Bertaux, J. L., et al. 1999, A\&A, 349, 660
Cox, D. P. 1998, Lecture Notes in Physics (Berlin: Springer Verlag), 506, 121

Cox, D., \& Helenius, L. 2003, ApJ, 583, 205

Cravens, T. E. 1997, GRL, 24, 105

Cravens, T. E. 2000, ApJ, 532, L153

Cravens, T. E., Robertson, I. P., \& Snowden, S. L. 2001, JGR, 106, 24883

Dennerl, K., Englhauser, J., \& Trumper, J. 1997, Science, 277, 1625

Egger, R. J., \& Aschenbach, B. 1995, A\&A, 294, L25

Freyberg, M. J. 1994, Ph.D. Thesis, Technishe Univ., München

Burrows, D. N., \& Guo, Z. 1998, Lecture Notes in Physics (Berlin: Springer Verlag), 506, 279

Haberli, R. M., Gombosi, T. I., De Zeeuw, D. L., Combi, M. R., \& Powell, K. G. 1997, Science, 276, 939

Hurwitz, M., \& Sholl, M. 1999, BAAS, 195, 8806

Jenkins, E. B. 2002, ApJ, 580, 938

Kharchenko, V., \& Dalgarno, A. 2000, JGR, 105, 18351

Krasnopolsky, V. 1997, Icarus, 128, 368

Kuntz, K. D., \& Snowden, S. 2000, ApJ, 543, 195

Lallement, R. 1998, Lecture Notes in Physics (Berlin: Springer Verlag), 506, 19

Lallement, R. 1999, Solar Wind Nine, AIP, 471, 205

Lallement, R., Raymond, J. C., \& Vallerga, J. V. 2003a, Adv. Space Res., in press

Lallement, R., Raymond, J. C., Bertaux, J.-L., et al. 2003b, A\&A, submitted

Lallement, R., Welsh, B., Vergely, J. L., Crifo, F., \& Sfeir, D. 2003c, $\mathrm{A} \& \mathrm{~A}$, in press

Lisse, C. M., Dennerl, K., Englhauser, J., et al. 1996, Science, 274, 205

McCammon, D., Almy, R., Apodaca, E., et al. 2002, ApJ, 576, 188

Mc Mullin, D. R., Judge, D. L., Phillips, E., et al. 2002, From solar min to solar max, ESA SP-508, 489

Mebold, U., Kerp, J., \& Kalberla, P. M. W. 1998, Lecture Notes in Physics (Berlin: Springer Verlag), 506, 199

Oegerle, W., Jenkins, E. B., Shelton, R. L., et al. 2000, BAAS, 32, 1404

Raymond, J., \& Smith, B. W. 1977, ApJS, 35, 419

Robertson, I., Cravens, T., Snowden, S., \& Linde, T. 2001, Sp. Sci. Rev., 97, 401

Rucinski, D., \& Fahr, H. J. 1989, A\&A, 224, 290

Sfeir, D., Lallement, R., Crifo, F., \& Welsh, B. Y. 1999, A\&A, 346, 785

Schwadron, N., \& Cravens, T. E. 2000, ApJ, 544, 558

Snowden, S., McCammon, D., Burrows, D. N., \& Mendenhall, J. A. 1994, ApJ, 424, 714

Snowden, S., Freyberg, M. J., Plucinsky, P. P., et al. 1995, ApJ, 454, 643

Snowden, S., Egger, R., Finkbeiner, D. P., Freyberg, M. J., \& Plucinsky, P. P. 1998, ApJ, 493, 715

Snowden, S., Freyberg, M., Kuntz, K., \& Sanders, W. 2000, ApJS, 128,171

Snowden, S. 2003, private commmunication

Vallerga, J. V., \& Slavin, J. 1998, Lecture Notes in Physics (Berlin: Springer Verlag), 506, 79

Wegmann, R., Schmidt, H. U., Lisse, C. M., Dennerl, K., \& Englhauser, J. 1998, Planet. Space Sci., 46, 603

Welsh, B., Sfeir, D., Sirk, M. M., \& Lallement, R. 1999, A\&A, 352, 308

Witte, M., et al. 2003, Adv. Space Res., in press

Wolff, B., Koester, D., \& Lallement, R. 1999, A\&A, 346, 969 